\title{
Sobolev Embeddings for Generalized Riesz Potentials of Functions in Morrey Spaces $L^{(1, \varphi)}(G)$ over Nondoubling Measure Spaces
}

\author{
Yoshihiro Sawano ${ }^{1}$ and Tetsu Shimomura ${ }^{2}$ \\ ${ }^{1}$ Department of Mathematics and Information Sciences, Tokyo Metropolitan University, Minami-Ohsawa 1-1, \\ Hachioji-shi, Tokyo 192-0397, Japan \\ ${ }^{2}$ Department of Mathematics, Graduate School of Education, Hiroshima University, Higashi-Hiroshima 739-8524, Japan
}

Correspondence should be addressed to Yoshihiro Sawano; yoshihiro-sawano@celery.ocn.ne.jp

Received 16 December 2012; Accepted 12 February 2013

Academic Editor: Alfonso Montes-Rodriguez

Copyright (c) 2013 Y. Sawano and T. Shimomura. This is an open access article distributed under the Creative Commons Attribution License, which permits unrestricted use, distribution, and reproduction in any medium, provided the original work is properly cited.

Our aim in this paper is to deal with the Sobolev embeddings for generalized Riesz potentials of functions in Morrey spaces $L^{(1, \varphi)}(G)$ over nondoubling measure spaces.

\section{Introduction}

In this paper, we show that many endpoint results about the Adams theorem still hold in the nondoubling setting and that the integral kernel can be generalized to a large extent. In [1], in the setting of the Lebesgue measure, for $0<\alpha<n$, recall that Adams considered and proved the boundedness of the fractional integral operator $I_{\alpha}$ given by

$$
I_{\alpha} f(x):=\int_{\mathbb{R}^{n}} \frac{f(y)}{|x-y|^{n-\alpha}} d y .
$$

The operator $I_{\alpha}$ is also called the fractional integral operator or the Riesz potential. We denote by $B(z, r)$ the ball $\left\{x \in \mathbb{R}^{n}\right.$ : $|x-z|<r\}$ with center $z$ and of radius $r>0$, and by $|B(z, r)|$ its Lebesgue measure, that is, $|B(z, r)|=\omega_{n} r^{n}$, where $\omega_{n}$ is the volume of the unit ball in $\mathbb{R}^{n}$. Let $G$ be a bounded open subset of $\mathbb{R}^{n}$. We denote its diameter by $d_{G}$;

$$
d_{G}=\sup \{|x-y|: x, y \in G\} .
$$
by

For $u \in L^{1}(G)$, we define the integral mean over $B(z, r)$

$$
u_{B(z, r)}=f_{B(z, r)} u(x) d x:=\frac{1}{|B(z, r)|} \int_{G \cap B(z, r)} u(x) d x .
$$

Let $1 \leq p<\infty$. If $\varphi$ is a positive function on the interval $(0, \infty)$ satisfying the doubling condition (see $(23))$, then we define the Morrey space $L^{(p, \varphi)}(G)$ to be the family of all $f \in$ $L_{\text {loc }}^{p}(G)$ for which there is a positive constant $C$ such that

$$
\begin{aligned}
& f_{B(z, r)}|f(x)|^{p} d x \\
& \quad \leq C^{p} \varphi(r) \quad \text { whenever } z \in G, 0<r \leq d_{G} .
\end{aligned}
$$

The norm of $f \in L^{(p, \varphi)}(G)$ is defined by the infimum of the constants $C$ satisfying the inequality above. When $\varphi(r) \equiv$ $r^{-\lambda}(r>0), L^{(p, \varphi)}(G)$ is denoted by $L_{p, \lambda}(G)$.

A direct consequence of this notation is that

$$
L_{p, \lambda p}(G) \supset L^{n / \lambda}(G)
$$

for $0<\lambda \leq n$ and $p \in[1, n / \lambda)$.

Some prefer to use the notation

$$
\|f\|_{M_{p, w(\cdot)}}=\sup _{r>0} w(r)\|f\|_{L^{p}(B(z, r))}
$$

with

$$
w(r)=\left(\omega_{n} r^{n}\right)^{-1 / p} \varphi(r)^{-1 / p}
$$

references [2-5]. 
Much about the case $p>1$ is known. Recall that the Adams theorem about the boundedness of fractional integral operators [1, Theorem 3.1] asserts that

$$
\left\|I_{\alpha} f\right\|_{L_{q, \lambda}} \leq C\|f\|_{L_{p, \lambda}},
$$

provided the parameters $p, q, \lambda$ satisfy

$$
0<\lambda \leq n, \quad 1<p<q<\infty, \quad \frac{1}{q}=\frac{1}{p}-\frac{\alpha}{\lambda} .
$$

See also research papers [2-4, 6-16] and a survey [5].

Meanwhile, only a few results are known for the case $p=1$. Trudinger [17, Theorem 1] proved that if $f \in L_{1,1}(G)=$ $L^{1}(G)$ then $\exp \left(a\left|I_{1} f\right|\right) \in L^{1}(G)$ for some constant $a>0$; this implies that the operator $I_{1}$ is bounded from $L_{1,1}(G)$ to $\exp \left(L^{1}\right)(G)$. See also Serrin [18] for an alternative proof. Recently, the boundedness of Riesz potentials from $L^{(1, \varphi)}(G)$ to Orlicz-Morrey spaces was shown in [19]. This result extends $[20,21]$. One of the reasons why the case when $p=1$ is difficult is the failure of the boundedness of the HardyLittlewood maximal operator $M$. In connection with this failure, we do not have Littlewood-Paley characterization. Due to these two difficulties, the case when $p=1$ is hard to analyze. However, from the point of PDEs, we are faced with analyzing the quantity

$$
\lim _{r \downarrow 0}\left(\sup _{x \in \mathbf{R}^{n}} \int_{B(x, r)} \frac{|V(y)|}{|x-y|^{n-\alpha}} d y\right)
$$

in connection of the Kato condition, where $V$ is the potential operator of the operator $-\Delta+V$. See [22, Section 2], for example. Consequently, despite the difficulty arising from harmonic analysis, the case when $p=1$ occurs naturally. As another evidence that the case when $p=1$ is of importance, we recall that the space $L_{1, \lambda}\left(\mathbb{R}^{n}\right)$ appears naturally in the following sharp maximal inequalities [23, Theorem 4.7], [24, Theorem 1.3], and [25, Theorem 1.2]: let $1<p<\infty$ and $\lambda \in(0, n]$. Then, there exists a constant $C>0$ such that

$$
\begin{aligned}
& C^{-1}\left(\left\|M^{\sharp} f\right\|_{L_{p, \lambda}}+\|f\|_{L_{1, \lambda}}\right) \\
& \quad \leq\|f\|_{L_{p, \lambda}} \leq C\left(\left\|M^{\sharp} f\right\|_{L_{p, \lambda}}+\|f\|_{L_{1, \lambda}}\right)
\end{aligned}
$$

for any measurable function $f$, where

$$
\begin{aligned}
M^{\sharp} f(x)= & \sup _{y \in \mathbb{R}^{n}, r>0} \frac{1}{|B(y, r)|} \\
& \times \int_{B(y, r)}\left|f(z)-\frac{1}{|B(y, r)|} \int_{B(y, r)} f(w) d w\right| d z
\end{aligned}
$$

is the sharp maximal operator due to Fefferman and Stein [26]. A disadvantage of using the Littlewood-Paley theory is that we lose the integrability of functions a little when we consider the inequality

$$
\sup _{j \in \mathbb{Z}}\left\|S_{j} f\right\|_{L_{1, \lambda}} \leq C\|f\|_{L_{1, \lambda}},
$$

where $\left\{S_{j}\right\}_{j=-\infty}^{\infty}$ is a Littlewood-Paley patch. By choosing a smooth function $\varphi \in C^{\infty}\left(\mathbb{R}^{n}\right)$ such that $\chi_{B(0,4) \backslash B(0,2)} \leq \varphi \leq$ $\chi_{B(0,8) \backslash B(0,1)}$, recall that we can define the $j$ th Littlewood-Paley patch by

$$
S_{j} f(x):=\mathscr{F}^{-1}\left[\varphi\left(2^{-j} \cdot\right) \cdot \mathscr{F} f\right](x)
$$

for $f \in \mathcal{S}^{\prime}\left(\mathbb{R}^{n}\right)$. Note that (13) is a direct consequence of the translation invariance of the space $L_{1, \lambda}\left(\mathbb{R}^{n}\right)$. But this loss caused by (13) is quite big. Note that

$$
\|f\|_{L_{1, \lambda}} \leq C \sup _{j \in \mathbb{Z}}\left\|S_{j} f\right\|_{L_{1, \lambda}}
$$

fails. See the appendix for a proof. When $p>1$, an approach using the Littlewood-Paley patch is taken effectively [27]. Indeed,

$$
C^{-1}\|f\|_{L_{p, \lambda}} \leq\left\|\left(\sum_{j=-\infty}^{\infty}\left|S_{j} f\right|^{2}\right)^{1 / 2}\right\|_{L_{p, \lambda}} \leq C\|f\|_{L_{p, \lambda}}
$$

for all $f \in L_{p, \lambda}\left(\mathbb{R}^{n}\right)$. However, for the case when $p=1$, due to the fact that the estimate (13) is essential when we consider the Littlewood-Paley patch, we prefer to avoid the LittlewoodPaley patch. See [28-43] for a huge amount of culmination of this approach.

Instead of using the Littlewood-Paley patch, we still have a good approach for the case when $p=1$. Just make a closer look at the integral kernel. Our method being simple enough, there is no need to stick to the geometric structure of $\mathbb{R}^{n}$. Our result relies completely only upon the positivity of the integral kernel. So, here and below, we work on a separable metric space $X$ equipped with a nonnegative Radon measure $\mu$, where we do not postulate any other condition on $\mu$. By $B(x, r)$, we denote the open ball centered at $x$ of radius $r>0$. While, given a point $p_{1}$ and $p_{2}$ in $\mathbb{R}^{n}$, we write $\left|p_{1}-p_{2}\right|$ for the distance of the points $p_{1}$ and $p_{2}$, and we write $d(x, y)$ for the distance of the points $x$ and $y$ in $X$. We assume that $\mu(\{x\})=0$ and that $0<\mu(B(x, r))<\infty$ for $x \in X$ and $r>0$ for simplicity. In the present paper, we do not postulate on $\mu$ the "so-called" doubling condition. Recall that a Radon measure $\mu$ is said to be doubling, if there exists a constant $C>0$ such that

$$
\mu(B(x, 2 r)) \leq C \mu(B(x, r))
$$

for all $x \in \operatorname{supp}(\mu)(=X)$ and $r>0$. Otherwise $\mu$ is said to be nondoubling. In connection with the $5 r$-covering lemma, the doubling condition had been a key condition in harmonic analysis.

Our aim in this paper is to show that, for the case $p=$ 1 , the operator $I_{\alpha}$ and its generalization $I_{\rho}$ are bounded from Morrey spaces $L^{(1, \varphi)}$ to Orlicz-Morrey spaces, or, to generalized Hölder spaces, whose definitions will be given in the next section, in the nondoubling setting. Our result extends the results in [17-21]. The definition of $I_{\rho}$ is the following: let $\rho$ be a function from $(0, \infty)$ to itself and satisfy

$$
\int_{0}^{r} \frac{\rho(t)}{t} d t<+\infty
$$


for all sufficiently small $r>0$. We do not have to postulate the doubling condition on $\rho$. See Remark 3 for an example which fails the doubling condition. We define

$$
I_{\rho} f(x)=\int_{G} \frac{\rho(d(x, y))}{\mu(B(x, 4 d(x, y)))} f(y) d \mu(y),
$$

where $f \in L^{1}(G)$. Instead of using

$$
I_{\rho}^{\dagger} f(x)=\int_{G} \frac{\rho(d(x, y))}{\mu(B(x, d(x, y)))} f(y) d \mu(y),
$$

we discuss $I_{\rho}$ defined above. This modification will be necessary in Lemma 9 for example. An example in [44, Section 2] shows that $I_{\rho}^{\dagger}$ is less likely to be bounded in general, although there does not exist a proof. We refer to [45] for an attempt of definining fractional integral operators by using the underlying measure $\mu$.

Note that (18) is necessary in order that the image by $I_{\rho}$ of $\chi_{B(x, r)}$, the indicator functions of the balls, belongs to $L^{p, \varphi}(G)$ at least when $\mu$ is the Lebesgue measure. Indeed, if

$$
\int_{0}^{r} \frac{\rho(t)}{t} d t=\infty
$$

for any sufficiently small $r>0$. Then, for $y \in B(x, r / 2)$ such that $B(x, r) \subset G$, we have

$$
\begin{aligned}
I_{\rho} \chi_{B(x, r)}(y) & =\int_{B(x, r)} \frac{\rho(|y-z|)}{|B(y, 4|y-z|)|} d z \\
& \geq \int_{B(y, r / 2)} \frac{\rho(|y-z|)}{|B(y, 4|y-z|)|} d z \\
& =C \int_{0}^{r / 2} \frac{\rho(t)}{t} d t \\
& =\infty
\end{aligned}
$$

by using the spherical coordinate.

We organize the remaining part of the present paper as follows. In Section 2, we set up some notations. Section 3 is devoted to stating our main results fully based on the notations in Section 2. Some auxiliary lemmas are collected in Section 4. Finally, theorems in the present paper are proven in Section 5.

\section{Notation and Terminologies}

Let $\mathscr{G}$ be the set of all continuous functions from $(0, \infty)$ to itself with the doubling condition, that is, there exists a constant $c_{\varphi} \geq 1$ such that

$$
\frac{1}{c_{\varphi}} \leq \frac{\varphi(r)}{\varphi(s)} \leq c_{\varphi} \quad \text { for } r, s>0 \text { with } \frac{1}{2} \leq \frac{r}{s} \leq 2 .
$$

We call the smallest number $c_{\varphi}$ satisfying (23) the doubling constant of $\varphi$. Note that in view of [46, page 445] and [47,
(1.2)], the doubling condition on $\varphi$ is a natural one. For $\varphi \in \mathscr{G}$, we define the Morrey space $L^{(1, \varphi)}(G)$ as follows:

$$
L^{(1, \varphi)}(G):=\left\{f \in L_{\mathrm{loc}}^{1}(G):\|f\|_{L^{(1, \varphi)}(G)}<\infty\right\}
$$

with the norm

$$
\begin{aligned}
\|f\|_{L^{(1, \varphi)}(G)}= & \sup _{z \in G, 0<r \leq d_{G}} \frac{1}{\varphi(r)} \\
& \cdot \frac{1}{\mu(B(z, 2 r))} \int_{B(z, r)}|f(x)| d \mu(x) .
\end{aligned}
$$

Then, a routine argument shows that $L^{(1, \varphi)}(G)$ is a Banach space. Due to the fact that $\mathbb{R}^{n}$ is a geometrically doubling space, we can prove that

$$
\begin{aligned}
C^{-1}\|f\|_{L^{(1, \varphi)}(G)} \leq & \sup _{z \in G, 0<r \leq d_{G}} \frac{1}{\varphi(r)} \\
& \cdot \frac{1}{\mu(B(z, k r))} \int_{B(z, r)}|f(x)| d \mu(x) \\
\leq & C\|f\|_{L^{(1, \varphi)}(G)}
\end{aligned}
$$

for all $k>1$. See [48, Proposition 1.1] for a technique used to prove this inequality. Note here that if $\varphi_{1}, \varphi_{2} \in \mathscr{G}$ and $\varphi_{1} / \varphi_{2}$ is bounded above on $\left(0, d_{G}\right)$, then

$$
L^{\left(1, \varphi_{1}\right)}(G) \subset L^{\left(1, \varphi_{2}\right)}(G),
$$

in particular, if there exists a constant $C \geq 1$ such that $C^{-1} \varphi_{1}(r) \leq \varphi_{2}(r) \leq C \varphi_{1}(r)$ for all $r>0$, then

$$
L^{\left(1, \varphi_{1}\right)}(G)=L^{\left(1, \varphi_{2}\right)}(G)
$$

with equivalent norms. A ball testing shows the following.

Proposition 1. The function $\varphi_{1} / \varphi_{2}$ is bounded above on $\left(0, d_{G}\right)$ if $L^{\left(1, \varphi_{1}\right)}(G) \subset L^{\left(1, \varphi_{2}\right)}(G)$ when $\mu=d x$.

Here and below, we write $A \lesssim B$ to indicate that there exists a constant $C$ independent of Morrey functions such that $A \leq C B$. The symbol $A \sim B$ stands for $A \lesssim B \lesssim A$.

Proof. According to [49, Proposition A], for any ball $B\left(x_{0}, r\right)$ contained in $G$, we have

$$
\left\|\chi_{B\left(x_{0}, r\right)}\right\|_{L^{\left(1, \varphi_{1}\right)}(G)} \sim \frac{1}{\varphi_{1}(r)}, \quad\left\|\chi_{B\left(x_{0}, r\right)}\right\|_{L^{\left(1, \varphi_{2}\right)}(G)} \sim \frac{1}{\varphi_{2}(r)} .
$$

If $L^{\left(1, \varphi_{1}\right)}(G) \subset L^{\left(1, \varphi_{2}\right)}(G)$, in the sense of sets, then by the closed graph theorem and the doubling condition on $\varphi_{1}$ and $\varphi_{2}$, we conclude

$$
\|f\|_{L^{\left(1, \varphi_{2}\right)}(G)} \leq C\|f\|_{L^{\left(1, \varphi_{1}\right)}(G)} .
$$

If we combine (29) and (30), then we obtain that $\varphi_{1} / \varphi_{2}$ is bounded above on $\left(0, d_{G}\right)$. 
Let us consider the family $\mathcal{y}$ of all continuous, increasing, convex, and bijective functions from $[0, \infty)$ to itself. For $\Phi \epsilon$ $\mathcal{Y}$, the Orlicz space $L^{\Phi}(G)$ is defined by

$$
L^{\Phi}(G):=\left\{f \in L_{\mathrm{loc}}^{1}(G):\|f\|_{L^{\Phi}(G)}<\infty\right\}
$$

where

$$
\|f\|_{L^{\Phi}(G)}:=\inf \left\{\lambda>0: \int_{G} \Phi\left(\frac{|f(x)|}{\lambda}\right) d \mu(x) \leq 1\right\} .
$$

If $\Phi_{1}, \Phi_{2} \in \mathcal{Y}$ are equivalent in the sense that there exists a constant $C \geq 1$ with

$$
\Phi_{1}\left(C^{-1} r\right) \leq \Phi_{2}(r) \leq \Phi_{1}(C r)
$$

for all $r>0$, then we see easily that

$$
L^{\Phi_{1}}(G)=L^{\Phi_{2}}(G)
$$

with equivalent norms. If

$$
\begin{gathered}
\Phi(r)=\exp \left(r^{p}\right), \quad \exp \left(\exp \left(r^{p}\right)\right), \\
r^{p}(\log r)^{\lambda} \quad \text { or } \quad r^{p}(\log r)^{q}(\log (\log r))^{\lambda} \quad(r>0)
\end{gathered}
$$

for large $r>0$, then $L^{\Phi}(G)$ will be denoted by

$$
\begin{gathered}
\exp \left(L^{p}\right)(G), \quad \exp \exp \left(L^{p}\right)(G), \\
L^{p}(\log L)^{\lambda}(G) \quad \text { or } \quad L^{p}(\log L)^{q}(\log \log L)^{\lambda}(G),
\end{gathered}
$$

respectively.

For $\Phi \in \mathcal{Y}$ and $\varphi \in \mathscr{G}$, the Orlicz-Morrey space $L^{(\Phi, \varphi)}(G)$ is defined by

$$
L^{(\Phi, \varphi)}(G):=\left\{f \in L_{\mathrm{loc}}^{1}(G):\|f\|_{L^{(\Phi, \varphi)}(G)}<\infty\right\},
$$

where

$$
\begin{aligned}
& \|f\|_{L^{(\Phi, \varphi)}(G)} \\
& :=\sup _{z \in G, 0<r \leq d_{G}} \inf \left\{\lambda>0: \frac{1}{\mu(B(z, 2 r))}\right. \\
& \left.\quad \times \int_{B(z, r)} \Phi\left(\frac{|f(x)|}{\lambda}\right) d \mu(x) \leq \varphi(r)\right\}
\end{aligned}
$$

(see $[50,51])$. Then, again it is routine to prove that $\|\cdot\|_{L^{(\Phi, \varphi)}(G)}$ is a norm and that $L^{(\Phi, \varphi)}(G)$ is a Banach space. Note that the space $L^{\Phi}$ is a special case of Orlicz-Morrey spaces when $\mu=d x$.

For $\varphi \in \mathscr{G}$ such that $\varphi$ is bounded, the generalized Hölder space is defined by

$$
\Lambda_{\varphi}(G)=\left\{f:\|f\|_{\Lambda_{\varphi}(G)}<\infty\right\}
$$

where

$$
\|f\|_{\Lambda_{\varphi}(G)}=\sup _{x, y \in G, x \neq y} \frac{|f(x)-f(y)|}{\varphi(2 d(x, y))} .
$$

Then, $\|f\|_{\Lambda_{\varphi}(G)}$ is a norm modulo constants and thereby $\Lambda_{\varphi}(G)$ is a Banach space. Since $\varphi$ is bounded, every $f \in$ $\Lambda_{\varphi}(G)$ is bounded. If $\varphi(r) \rightarrow 0$ as $r \downarrow 0$, then every $f \in$ $\Lambda_{\varphi}(G)$ is continuous. For details, we refer to [52].

\section{Main Results}

In this section, we state our main theorems, whose proofs are given in Section 5.

Throughout this paper, let $G$ be a bounded open set in $X$ and denote by $c_{\varphi}$, the doubling constant of $\varphi \in \mathscr{G}$.

Let us begin with the following result, which is the one of Gunawan type [9].

Theorem 2. Let $\rho:(0, \infty) \rightarrow(0, \infty)$ be a measurable function such that there exist $k_{1}, k_{2}, C_{\rho}$ such that

$$
\begin{gathered}
0<16 k_{1} \leq 1 \leq k_{2}<\infty \\
\sup _{r / 2 \leq s \leq r} \rho(s) \leq C_{\rho} \int_{k_{1} r}^{k_{2} r} \frac{\rho(s)}{s} d s \quad(r>0) .
\end{gathered}
$$

Let $\varphi \in \mathscr{G}$, and define

$$
\psi(r):=\left(\int_{0}^{4 k_{2} r} \frac{\rho(t)}{t} d t\right) \varphi(r)+\int_{2 k_{1} r}^{4 k_{2} d_{G}} \frac{\rho(t) \varphi(t)}{t} d t
$$

for $0<r \leq d_{G}$. Then, there exists a constant $C>0$ such that

$$
\frac{1}{\mu(B(z, 4 r))} \int_{B(z, r)}\left|I_{\rho} f(x)\right| d \mu(x) \leq C \psi(r)\|f\|_{L^{(1, \varphi)}(G)}
$$

for $z \in G, 0<r \leq d_{G}$ and $f \in L^{(1, \varphi)}(G)$, where $C>0$ is a constant depending only on $C_{\rho}, c_{\varphi}, k_{1}$, and $k_{2}$.

Remark 3. (1) Here it is not significant for us to choose 16; it counts that any number will do as long as it is small enough.

(2) The number 4 in the right-hand side seems to be essential. According to [44, Section 2], it can happen that the norms

$$
\begin{aligned}
& \sup _{z \in G, 0<r \leq d_{G}}\left(\frac{r^{\nu}}{\mu(B(z, 4 r))} \int_{B(z, r)}|f(w)|^{p} d \mu(w)\right)^{1 / p}, \\
& \sup _{z \in G, 0<r \leq d_{G}}\left(\frac{r^{\nu}}{\mu(B(z, 2 r))} \int_{B(z, r)}|f(w)|^{p} d \mu(w)\right)^{1 / p}
\end{aligned}
$$

are not equivalent for $1 \leq p<\infty$.

(3) In view of [53, Lemma 2.5], we see that $(1-\Delta)^{-\alpha / 2}$ falls under the scope of Theorem 2. Indeed, Nagayasu and Wadade showed that the kernel $\rho$ which corresponds to $(1-\Delta)^{-\alpha / 2}$ satisfies

$$
\rho(r) \sim r^{\alpha} \quad(0<r<1), \quad \rho(r) \lesssim e^{-r} \quad(r \geq 1) .
$$


This means that we have (41) with $k_{1}=1 / 16$ and $k_{2}=1$. Note that $\rho \in \mathscr{G}$ implies (41). See also [54, Remark 2.2].

Remark 4. Theorem 2 is proved in [19] when $G=\mathbb{R}^{n}$ and $\rho \in \mathscr{G}$. See also [21].

We now state a result for Orlicz-Morrey spaces.

Theorem 5. Let $\rho, \tilde{\rho}:(0, \infty) \rightarrow(0, \infty)$ be measurable functions such that there exist $k_{1}, k_{2}, C_{\rho}$ such that $0<16 k_{1} \leq 1 \leq$ $k_{2}<\infty$ and that

$$
\begin{gathered}
\sup _{r / 2 \leq s \leq r} \rho(s) \leq C_{\rho} \int_{k_{1} r}^{k_{2} r} \frac{\rho(s)}{s} d s, \\
\sup _{r / 2 \leq s \leq r} \tilde{\rho}(s) \leq C_{\widetilde{\rho}} \int_{k_{1} r}^{k_{2} r} \frac{\widetilde{\rho}(s)}{s} d s \quad(r>0) .
\end{gathered}
$$

Let $\varphi \in \mathscr{G}$. Assume

$$
\int_{0}^{1} \frac{\rho(t) \varphi(t)}{t} d t=\infty
$$

and that $\tilde{\rho} / \rho$ is continuous and decreasing.

Define

$$
\begin{aligned}
& \psi_{1}(r):=\int_{2 k_{1} r}^{4 k_{2} d_{G}} \frac{\rho(t) \varphi(t)}{t} d t, \\
& \kappa(r):=\frac{\psi_{1}(r) \tilde{\rho}\left(4 k_{2} r\right)}{\rho\left(4 k_{2} r\right)}, \\
& \psi(r):=\left(\int_{0}^{2 k_{2} r} \frac{\tilde{\rho}(t)}{t} d t\right) \varphi(r)+\int_{2 k_{1} r}^{4 k_{2} d_{G}} \frac{\tilde{\rho}(t) \varphi(t)}{t} d t
\end{aligned}
$$

for $0<r \leq d_{G}$. If $\Phi \in \mathcal{Y}$ satisfies

$$
C_{G}=\sup \left\{\frac{\left(\psi_{1} \circ \kappa^{-1}\right)(s)}{\Phi^{-1}(s)}: \kappa\left(d_{G}\right) \leq s<\infty\right\}<\infty,
$$

then there exists a constant $A>0$ such that

$$
\frac{1}{\mu(B(z, 4 r))} \int_{B(z, r)} \Phi\left(\frac{\left|I_{\rho} f(x)\right|}{A\|f\|_{L^{(1, \varphi)}(G)}}\right) d \mu(x) \leq \psi(r)
$$

for $z \in G, 0<r \leq d_{G}$ and $f \in L^{(1, \varphi)}(G)$, where $A>0$ is a constant depending only on $C_{\rho}, C_{\tilde{\rho}}, c_{\varphi}, k_{1}, k_{2}$, and $C_{G}$.

Remark 6. Note that $\kappa$ is bijective from $\left(0, d_{G}\right]$ to $\left[\kappa\left(d_{G}\right), \infty\right)$ by the assumptions in the theorem. Indeed, by the definition of $\kappa$ above, $\kappa$ is a decreasing function. In addition, $\lim _{r \downarrow 0} \kappa(r)=\infty$, showing that $\kappa:\left(0, d_{G}\right] \rightarrow\left[\kappa\left(d_{G}\right), \infty\right)$ is bijective.

Finally, we shall show a result of Gunawan type about continuity.

Theorem 7. Let $\rho:(0, \infty) \rightarrow(0, \infty)$ be a measurable function such that there exist $k_{1}, k_{2}, C_{\rho}$ such that

$$
\begin{gathered}
0<16 k_{1} \leq 1 \leq k_{2}<\infty, \\
\sup _{r / 2 \leq s \leq r} \rho(s) \leq C_{\rho} \int_{k_{1} r}^{k_{2} r} \frac{\rho(s)}{s} d s \quad(r>0) .
\end{gathered}
$$

Let $\varphi \in \mathscr{G}$. Assume the following condition on $\rho$. There are $0<\theta \leq 1$ and $C_{\rho}^{\prime}>0$ such that

$$
\begin{gathered}
\left|\frac{\rho(d(x, y))}{\mu(B(x, 4 d(x, y)))}-\frac{\rho(d(z, y))}{\mu(B(z, 4 d(z, y)))}\right| \\
\leq C_{\rho}^{\prime}\left(\frac{d(x, z)}{d(x, y)}\right)^{\theta} \frac{\rho(d(x, y))}{\mu(B(x, 4 d(x, y)))}
\end{gathered}
$$

whenever $d(x, z) \leq d(x, y) / 2$. Assume in addition the Dini condition

$$
\int_{0}^{1} \frac{\rho(t) \varphi(t)}{t} d t<\infty
$$

If

$$
\begin{array}{r}
\psi(r)=\int_{0}^{3 k_{2} r} \frac{\rho(t) \varphi(t)}{t} d t+r^{\theta} \int_{2 k_{1} r}^{4 k_{2} d_{G}} \frac{\rho(t) \varphi(t)}{t^{1+\theta}} d t \\
\text { for } 0<r \leq d_{G},
\end{array}
$$

then $I_{\rho}$ is bounded from $L^{(1, \varphi)}(G)$ to $\Lambda_{\psi}(G)$. More precisely,

$$
\left\|I_{\rho} f\right\|_{\Lambda_{\psi}(G)} \leq C\|f\|_{L^{(1, \varphi)}(G)},
$$

where $C>0$ is a constant depending only on $C_{\rho}, C_{\rho}^{\prime}, c_{\varphi}, k_{1}, k_{2}$, and $\theta$.

Note that if $\int_{0}^{1}(\rho(t) \varphi(t) / t) d t<\infty$ and $0<\theta \leq 1$, then

$$
r \in\left(0, d_{G}\right] \longmapsto r^{\theta} \int_{2 k_{1} r}^{4 k_{2} d_{G}} \frac{\rho(t) \varphi(t)}{t^{1+\theta}} d t \in[0, \infty)
$$

is bounded.

\section{Preliminary Lemmas}

Lemma 8. Let $\rho:(0, \infty) \rightarrow(0, \infty)$ be a measurable function such that there exist $k_{1}, k_{2}, C_{\rho}$ such that

$$
\begin{gathered}
0<16 k_{1} \leq 1 \leq k_{2}<\infty \\
\sup _{r / 2 \leq s \leq r} \rho(s) \leq C_{\rho} \int_{k_{1} r}^{k_{2} r} \frac{\rho(s)}{s} d s \quad(r>0) .
\end{gathered}
$$

Let $\varphi \in \mathscr{G}$. Then

$$
\begin{aligned}
& \int_{B(x, r)} \frac{\rho(d(x, y))}{\mu(B(x, 4 d(x, y)))}|f(y)| d \mu(y) \\
& \leq C\left(\int_{0}^{2 k_{2} r} \frac{\rho(t) \varphi(t)}{t} d t\right)\|f\|_{L^{(1, \varphi)}(G)},
\end{aligned}
$$

where $C>0$ is a constant depending only on $C_{\rho}, c_{\varphi}, k_{1}$, and $k_{2}$. 
Moreover, if $k \geq 0$, then

$$
\begin{gathered}
\int_{B\left(x, d_{G}\right) \backslash B(x, r)} \frac{\rho(d(x, y))}{\mu(B(x, 4 d(x, y))) d(x, y)^{k}}|f(y)| d \mu(y) \\
\leq C\left(\int_{2 k_{1} r}^{4 k_{2} d_{G}} \frac{\rho(t) \varphi(t)}{t^{1+k}} d t\right)\|f\|_{L^{(1, \varphi)}(G)},
\end{gathered}
$$

where $C>0$ is a constant depending only on $C_{\rho}, c_{\varphi}, k_{1}, k_{2}$, and $k$.

Proof. If $y \in B\left(x, 2^{j} r\right) \backslash B\left(x, 2^{j-1} r\right)$ and $j \in \mathbb{Z}$, then a geometric observation shows

$$
\begin{aligned}
& \frac{\rho(d(x, y))}{\mu(B(x, 4 d(x, y))) d(x, y)^{k}} \\
& \leq \frac{1}{\mu\left(B\left(x, 2^{j+1} r\right)\right)\left(2^{j-1} r\right)^{k}} \sup _{2^{j-1} r \leq s \leq 2^{j} r} \rho(s) \\
& \leq \frac{C_{\rho}}{\mu\left(B\left(x, 2^{j+1} r\right)\right)\left(2^{j-1} r\right)^{k}} \int_{2^{j} k_{1} r}^{2^{j} k_{2} r} \frac{\rho(s)}{s} d s .
\end{aligned}
$$

Hence,

$$
\begin{aligned}
& \int_{B\left(x, 2^{j} r\right) \backslash B\left(x, 2^{j-1} r\right)} \frac{\rho(d(x, y))}{\mu(B(x, 4 d(x, y))) d(x, y)^{k}}|f(y)| d \mu(y) \\
& \leq \frac{C_{\rho}}{\left(2^{j-1} r\right)^{k}} \int_{2^{j} k_{1} r}^{2^{j} k_{2} r} \frac{\rho(s)}{s} d s \\
& \times \frac{1}{\mu\left(B\left(x, 2^{j+1} r\right)\right)} \int_{B\left(x, 2^{j} r\right)}|f(y)| d \mu(y) \\
&= C_{\rho} \frac{\varphi\left(2^{j} r\right)}{\left(2^{j-1} r\right)^{k}} \int_{2^{j} k_{1} r}^{2^{j} k_{2} r} \frac{\rho(s)}{s} d s \\
& \quad \times \frac{1}{\varphi\left(2^{j} r\right) \mu\left(B\left(x, 2^{j+1} r\right)\right)} \int_{B\left(x, 2^{j} r\right)}|f(y)| d \mu(y) \\
& \leq C_{\rho} \frac{\varphi\left(2^{j} r\right)}{\left(2^{j-1} r\right)^{k}} \int_{2^{j} k_{1} r}^{2^{j} k_{2} r} \frac{\rho(s)}{s} d s \times\|f\|_{L^{(1, \varphi)}(G)} .
\end{aligned}
$$

Set $d:=\left[1+\log _{2}\left(k_{2} / k_{1}\right)\right]$. Then, by virtue of the doubling condition on $\varphi$, we have

$$
\begin{aligned}
& \frac{\varphi\left(2^{j} r\right)}{\left(2^{j-1} r\right)^{k}} \int_{2^{j} k_{1} r}^{2^{j} k_{2} r} \frac{\rho(s)}{s} d s \\
& \leq 2^{k} \frac{\varphi\left(2^{j} r\right)}{\left(2^{j} r\right)^{k}} \int_{2^{j} k_{1} r}^{2^{j+d} k_{1} r} \frac{\rho(s)}{s} d s \\
& \quad=2^{k} \sum_{l=1}^{d} \frac{\varphi\left(2^{j} r\right)}{\left(2^{j} r\right)^{k}} \int_{2^{j+l-1} k_{1} r}^{2^{j+l} k_{1} r} \frac{\rho(s)}{s} d s
\end{aligned}
$$

$$
\begin{aligned}
& \leq C_{1}\left(2^{d+1} k_{1}\right)^{k} \sum_{l=1}^{d} \int_{2^{j+l-1} k_{1} r}^{2^{j+l} k_{1} r} \frac{\varphi(s)}{\left(2^{j+d} k_{1} r\right)^{k}} \cdot \frac{\rho(s)}{s} d s \\
& \leq C_{1}\left(2^{d+1} k_{1}\right)^{k} \sum_{l=1}^{d} \int_{2^{j+l-1} k_{1} r}^{2^{j+l} k_{1} r} \frac{\varphi(s) \rho(s)}{s^{1+k}} d s \\
& =C_{1}\left(2^{d+1} k_{1}\right)^{k} \int_{2^{j} k_{1} r}^{2^{j+d} k_{1} r} \frac{\varphi(s) \rho(s)}{s^{1+k}} d s,
\end{aligned}
$$

where $C_{1}>0$ is a constant depending only on $c_{\varphi}, k_{1}$, and $k_{2}$.

Consequently, since $\mu(\{x\})=0$,

$$
\begin{aligned}
& \int_{B(x, r)} \frac{\rho(d(x, y))}{\mu(B(x, 4 d(x, y)))}|f(y)| d \mu(y) \\
& =\sum_{j=0}^{\infty} \int_{B\left(x, 2^{-j} r\right) \backslash B\left(x, 2^{-j-1} r\right)} \frac{\rho(d(x, y))}{\mu(B(x, 4 d(x, y)))}|f(y)| d \mu(y) \\
& \leq \sum_{j=0}^{\infty} C_{\rho} C_{1} \int_{2^{-j} k_{1} r}^{2^{-j+d} k_{1} r} \frac{\varphi(s) \rho(s)}{s} d s \times\|f\|_{L^{(1, \varphi)}(G)} \\
& \leq C_{\rho} C_{1}\left(\int_{0}^{2 k_{2} r} \frac{\rho(t) \varphi(t)}{t} d t\right)\|f\|_{L^{(1, \varphi)}(G)},
\end{aligned}
$$

which proves (58).

We choose $j_{0} \in \mathbb{Z}$, so that $d_{G} \leq 2^{j_{0}} r<2 d_{G}$. Then, we have

$$
\begin{gathered}
\int_{B\left(x, d_{G}\right) \backslash B(x, r)} \frac{\rho(d(x, y))}{\mu(B(x, 4 d(x, y))) d(x, y)^{k}}|f(y)| d \mu(y) \\
\leq \sum_{j=1}^{j_{0}} \int_{B\left(x, 2^{j} r\right) \backslash B\left(x, 2^{j-1} r\right)} \frac{\rho(d(x, y))}{\mu(B(x, 4 d(x, y))) d(x, y)^{k}} \\
\times|f(y)| d \mu(y) \\
\leq C_{\rho} C_{1}\left(2^{d+1} k_{1}\right)^{k} \sum_{j=1}^{j_{0}} \int_{2^{j} k_{1} r}^{2^{j+d} k_{1} r} \frac{\varphi(s) \rho(s)}{s^{1+k}} d s \times\|f\|_{L^{(1, \varphi)}(G)} \\
\leq C_{\rho} C_{1}\left(2^{d+1} k_{1}\right)^{k}\left(\int_{2 k_{1} r}^{4 k_{2} d_{G}} \frac{\rho(t) \varphi(t)}{t^{1+k}} d t\right)\|f\|_{L^{(1, \varphi)}(G)} .
\end{gathered}
$$

Thus, since $k, k_{1}, d$ being constants, (59) follows.

Lemma 9. Let $\rho:(0, \infty) \rightarrow(0, \infty)$ be a measurable function such that there exist $k_{1}, k_{2}, C_{\rho}$ such that

$$
0<16 k_{1} \leq 1 \leq k_{2}<\infty \text {, }
$$

and that

$$
\sup _{r / 2 \leq s \leq r} \rho(s) \leq C_{\rho} \int_{k_{1} r}^{k_{2} r} \frac{\rho(s)}{s} d s \quad(r>0) .
$$


Then, for all $f \in L^{(1, \varphi)}(G)$,

$$
\begin{aligned}
& \frac{1}{\mu(B(z, 2 r))} \\
& \quad \times \int_{B(z, r)}\left(\int_{B(z, r)} \frac{\rho(d(x, y))}{\mu(B(x, 4 d(x, y)))}|f(y)| d \mu(y)\right) d \mu(x) \\
& \leq C \varphi(r)\left(\int_{0}^{2 k_{2} r} \frac{\rho(t)}{t} d t\right)\|f\|_{L^{(1, \varphi)}(G)} .
\end{aligned}
$$

Proof. By Fubini's theorem and the dyadic decomposition of the ball, we have

$$
\begin{aligned}
& \int_{B(z, r)}\left(\int_{B(z, r)} \frac{\rho(d(x, y))}{\mu(B(x, 4 d(x, y)))}|f(y)| d \mu(y)\right) d \mu(x) \\
& =\int_{B(z, r)}|f(y)| \\
& \quad \times\left(\int_{B(z, r)} \frac{\rho(d(x, y))}{\mu(B(x, 4 d(x, y)))} d \mu(x)\right) d \mu(y) \\
& \leq \int_{B(z, r)}|f(y)| \\
& \quad \times\left(\sum_{j=0}^{\infty} \int_{B\left(y, 2^{-j+1} r\right) \backslash B\left(y, 2^{-j} r\right)} \frac{\sup _{2^{-j} r \leq s \leq 2^{-j+1} r} \rho(s)}{\mu\left(B\left(x, 2^{-j+2} r\right)\right)}\right. \\
& \left.\leq \int_{B(z, r)}|f(y)| \quad \times d \mu(x)\right) d \mu(y) \\
& \quad \times\left(\sum_{j=0}^{\infty} \int_{\left.B\left(y, 2^{-j+1} r\right)\right)} \frac{\sup _{2^{-j} r \leq s \leq 2^{-j+1} r} \rho(s)}{\mu\left(B\left(y, 2^{-j+1} r\right)\right)}\right. \\
& \times d \mu(x)) d \mu(y) .
\end{aligned}
$$

Since $\rho$ satisfies (66), we have

$$
\begin{aligned}
& \int_{B(z, r)}\left(\int_{B(z, r)} \frac{\rho(d(x, y))}{\mu(B(x, 4 d(x, y)))}|f(y)| d \mu(y)\right) d \mu(x) \\
& \leq C_{\rho} \int_{B(z, r)}|f(y)|\left(\sum_{j=0}^{\infty} \int_{2^{-j+1} k_{1} r}^{2^{-j+1} k_{2} r} \frac{\rho(s)}{s} d t\right) d \mu(y) \\
& \leq C_{\rho}\left(\int_{0}^{2 k_{2} r} \frac{\rho(t)}{t} d t\right) \int_{B(z, r)}|f(y)| d \mu(y) \\
& \leq C_{\rho} \varphi(r) \mu(B(z, 2 r))\left(\int_{0}^{2 k_{2} r} \frac{\rho(t)}{t} d t\right)\|f\|_{L^{(1, \varphi)}(G)},
\end{aligned}
$$

as required.

\section{Proofs of the Theorems}

We are now ready to prove our theorems.

Proof of Theorem 2. Let $z \in G$ and $r \in\left(0, d_{G}\right]$. By the positivity of the kernel, we may assume that $f \geq 0$. We write

$$
\begin{aligned}
& \frac{1}{\mu(B(z, 4 r))} \int_{B(z, r)} I_{\rho} f(x) d \mu(x) \\
& \leq \frac{1}{\mu(B(z, 4 r))} \\
& \quad \times \int_{B(z, r)}\left(\int_{B(x, r)} \frac{\rho(d(x, y))}{\mu(B(x, 4 d(x, y)))}\right. \\
& \left.\quad+\frac{1}{\mu(B(z, 4 r))} \times f(y) d \mu(y)\right) d \mu(x) \\
& \quad \times \int_{B(z, r)}\left(\int_{B\left(x, d_{G}\right) \backslash B(x, r)} \frac{\rho(d(x, y))}{\mu(B(x, 4 d(x, y)))}\right. \\
& \left.\leq \frac{1}{\mu(B(z, 4 r))} \times f(y) d \mu(y)\right) d \mu(x) \\
& \quad \times \int_{B(z, 2 r)}\left(\int_{B(z, 2 r)} \frac{\rho(d(x, y))}{\mu(B(x, 4 d(x, y)))}\right. \\
& \quad+\frac{1}{\mu(B(z, 4 r))} \\
& \quad \times \int_{B(z, r)}\left(\int_{B\left(x, d_{G}\right) \backslash B(x, r)} \frac{\rho(d(x, y))}{\mu(B(x, 4 d(x, y)))}\right. \\
& \times f(y) d \mu(y)) d \mu(x)
\end{aligned}
$$

$$
=I_{1}+I_{2}
$$

for $z \in G$ and $0<r \leq d_{G}$. By Lemma 9, we have

$$
\begin{aligned}
I_{1} & \leq C_{1} \varphi(r)\left(\int_{0}^{4 k_{2} r} \frac{\rho(t)}{t} d t\right)\|f\|_{L^{(1, \varphi)}(G)} \\
& \leq C_{1} \psi(r)\|f\|_{L^{(1, \varphi)}(G)} .
\end{aligned}
$$

Meanwhile, by Lemma 8 we have

$$
\begin{aligned}
I_{2} & \leq C_{2}\left(\int_{2 k_{1} r}^{4 k_{2} d_{G}} \frac{\rho(t) \varphi(t)}{t} d t\right)\|f\|_{L^{(1, \varphi)}(G)} \\
& \leq C_{2} \psi(r)\|f\|_{L^{(1, \varphi)}(G)} .
\end{aligned}
$$


Hence, it follows from (71) and (72) that

$$
\frac{1}{\mu(B(z, 4 r))} \int_{B(z, r)} I_{\rho} f(x) d \mu(x) \leq C \psi(r)\|f\|_{L^{(1, \varphi)}(G)},
$$

where $C>0$ depends only on $C_{\rho}, c_{\varphi}, k_{1}$, and $k_{2}$.

Proof of Theorem 5. By Theorem 2, we have

$$
\frac{1}{\mu(B(z, 4 r))} \int_{B(z, r)}\left|I_{\tilde{\rho}} f(x)\right| d \mu(x) \leq C_{1} \psi(r)\|f\|_{L^{(1, \varphi)}(G)}
$$

for $z \in G$ and $0<r \leq d_{G}$.

Let $g:=|f| /\|f\|_{L^{(1, \varphi)}(G)}$. For $x \in G$ and $0<\delta \leq d_{G}$, since $\tilde{\rho} / \rho$ is decreasing, we have by Lemma 8

$$
\begin{aligned}
& I_{\rho} g(x) \\
& =\int_{B(x, \delta)} \frac{\rho(d(x, y))}{\mu(B(x, 4 d(x, y)))} g(y) d \mu(y) \\
& +\int_{B\left(x, d_{G}\right) \backslash B(x, \delta)} \frac{\rho(d(x, y))}{\mu(B(x, 4 d(x, y)))} g(y) d \mu(y) \\
& \leq \frac{\rho(\delta)}{\tilde{\rho}(\delta)} \int_{B(x, \delta)} \frac{\widetilde{\rho}(d(x, y))}{\mu(B(x, 4 d(x, y)))} g(y) d \mu(y) \\
& +C_{2} \int_{2 k_{1} \delta}^{4 k_{2} d_{G}} \frac{\rho(t) \varphi(t)}{t} d t \\
& \leq \frac{\rho(\delta)}{\widetilde{\rho}(\delta)} I_{\widetilde{\rho}} g(x)+C_{2} \psi_{1}(\delta) \\
& \leq \frac{\rho\left(4 k_{2} \delta\right)}{\widetilde{\rho}\left(4 k_{2} \delta\right)} I_{\widetilde{\rho}} g(x)+C_{2} \psi_{1}(\delta) \text {. }
\end{aligned}
$$

Hence, in view of the definition of $\kappa$, we have

$$
I_{\rho} g(x) \leq \frac{\psi_{1}(\delta)}{\kappa(\delta)} I_{\widetilde{\rho}} g(x)+C_{2} \psi_{1}(\delta)
$$

Now let

$$
\delta:= \begin{cases}\kappa^{-1}\left(I_{\tilde{\rho}} g(x)\right) & \text { when } I_{\tilde{\rho}} g(x) \geq \kappa\left(d_{G}\right), \\ d_{G} & \text { when } I_{\tilde{\rho}} g(x)<\kappa\left(d_{G}\right) .\end{cases}
$$

Observe that

$$
\psi_{1}(\delta)= \begin{cases}\psi_{1}\left(\kappa^{-1}\left(I_{\widetilde{\rho}} g(x)\right)\right) & \text { when } I_{\widetilde{\rho}} g(x) \geq \kappa\left(d_{G}\right), \\ \psi_{1}\left(d_{G}\right) & \text { when } I_{\widetilde{\rho}} g(x)<\kappa\left(d_{G}\right),\end{cases}
$$

by definition.

$$
\begin{aligned}
& \text { We claim that } \\
& \frac{\psi_{1}(\delta)}{\kappa(\delta)} I_{\tilde{\rho}} g(x) \\
& \leq \begin{cases}\psi_{1}\left(\kappa^{-1}\left(I_{\tilde{\rho}} g(x)\right)\right) & \text { when } I_{\tilde{\rho}} g(x) \geq \kappa\left(d_{G}\right), \\
\psi_{1}\left(d_{G}\right) & \text { when } I_{\tilde{\rho}} g(x)<\kappa\left(d_{G}\right) .\end{cases}
\end{aligned}
$$

Indeed, when $I_{\widetilde{\rho}} g(x)<\kappa\left(d_{G}\right)$, we have $\delta=d_{G}$. Hence,

$$
\frac{\psi_{1}(\delta)}{\kappa(\delta)} I_{\widetilde{\rho}} g(x)=\psi_{1}\left(d_{G}\right) \times \frac{1}{\kappa\left(d_{G}\right)} I_{\tilde{\rho}} g(x) \leq \psi_{1}\left(d_{G}\right)
$$

When $I_{\tilde{\rho}} g(x) \geq \kappa\left(d_{G}\right)$, we have $\delta=\kappa^{-1}\left(I_{\tilde{\rho}} g(x)\right)$. Hence,

$$
\begin{aligned}
\frac{\psi_{1}(\delta)}{\kappa(\delta)} I_{\tilde{\rho}} g(x) & =\frac{\psi_{1}\left(\kappa^{-1}\left(I_{\tilde{\rho}} g(x)\right)\right)}{I_{\tilde{\rho}} g(x)} I_{\tilde{\rho}} g(x) \\
& =\psi_{1}\left(\kappa^{-1}\left(I_{\tilde{\rho}} g(x)\right)\right) .
\end{aligned}
$$

Consequently our claim (79) is justified.

$$
\text { It follows from (76) and (79) that }
$$

$$
I_{\rho} g(x) \leq\left(1+C_{2}\right) \max \left\{\psi_{1}\left(\kappa^{-1}\left(I_{\tilde{\rho}} g(x)\right)\right), \psi_{1}\left(d_{G}\right)\right\}
$$

By (49), we obtain

$$
\left(\psi_{1} \circ \kappa^{-1}\right)(s) \leq C_{G} \Phi^{-1}(s) \quad \text { for } \kappa\left(d_{G}\right) \leq s<\infty
$$

Hence, taking $A:=C_{G}\left(C_{1}+1\right)\left(1+C_{2}\right)$, we establish

$$
\begin{aligned}
& \frac{\left|I_{\rho} f(x)\right|}{A\|f\|_{L^{(1, \varphi)}(G)}} \\
& \quad \leq \frac{I_{\rho} g(x)}{A} \\
& \quad \leq \frac{\max \left\{\psi_{1}\left(\kappa^{-1}\left(I_{\tilde{\rho}} g(x)\right)\right), \psi_{1}\left(d_{G}\right)\right\}}{C_{G}\left(C_{1}+1\right)} \\
& \quad=\frac{\max \left\{\psi_{1}\left(\kappa^{-1}\left(I_{\tilde{\rho}} g(x)\right)\right), \psi_{1}\left(\kappa^{-1}\left(\kappa\left(d_{G}\right)\right)\right)\right\}}{C_{G}\left(C_{1}+1\right)} \\
& \quad \leq \frac{\max \left\{\Phi^{-1}\left(I_{\tilde{\rho}} g(x)\right), \Phi^{-1}\left(\kappa\left(d_{G}\right)\right)\right\}}{C_{1}+1} .
\end{aligned}
$$

Since $\tilde{\rho} / \rho$ is decreasing and

$$
\left(\widetilde{\rho}\left(4 k_{2} d_{G}\right) / \rho\left(4 k_{2} d_{G}\right)\right) \psi_{1}\left(d_{G}\right)=\kappa\left(d_{G}\right),
$$

we see that

$$
\begin{aligned}
\psi(r) & \geq \int_{2 k_{1} d_{G}}^{4 k_{2} d_{G}} \frac{\widetilde{\rho}(t) \varphi(t)}{t} d t \\
& \geq \frac{\widetilde{\rho}\left(4 k_{2} d_{G}\right)}{\rho\left(4 k_{2} d_{G}\right)} \int_{2 k_{1} d_{G}}^{4 k_{2} d_{G}} \frac{\rho(t) \varphi(t)}{t} d t \\
& =\kappa\left(d_{G}\right)
\end{aligned}
$$


for all $0<r \leq d_{G}$. Hence, with the aid of (74), we have

$$
\begin{aligned}
& \frac{1}{\mu(B(z, 4 r))} \int_{B(z, r)} \Phi\left(\frac{\left|I_{\rho} f(x)\right|}{A\|f\|_{L^{(1, \varphi)}(G)}}\right) d \mu(x) \\
& \leq \frac{1}{C_{1}+1} \\
& \times \frac{1}{\mu(B(z, 4 r))} \int_{B(z, r)} \max \left\{I_{\tilde{\rho}} g(x), \kappa\left(d_{G}\right)\right\} d \mu(x) \\
& \leq \frac{1}{C_{1}+1} \\
& \times\left(\frac{1}{\mu(B(z, 4 r))} \int_{B(z, r)} I_{\tilde{\rho}} g(x) d \mu(x)\right. \\
& \leq \frac{1}{C_{1}+1}\left(C_{1} \psi(r)+\psi(r)\right)=\psi(r),
\end{aligned}
$$

which proves (50).

Proof of Theorem 7. Write

$$
\begin{aligned}
& I_{\rho} f(x)-\mathrm{I}_{\rho} f(z) \\
& =\int_{B(x, 2 d(x, z))} \frac{\rho(d(x, y))}{\mu(B(x, 4 d(x, y)))} f(y) d \mu(y) \\
& \quad-\int_{B(x, 2 d(x, z))} \frac{\rho(d(z, y))}{\mu(B(z, 4 d(z, y)))} f(y) d \mu(y) \\
& +\int_{G \backslash B(x, 2 d(x, z))}\left(\frac{\rho(d(x, y))}{\mu(B(x, 4 d(x, y)))}\right. \\
& \times f(y) d \mu(y) .
\end{aligned}
$$

By (58), we have

$$
\begin{aligned}
& \int_{B(x, 2 d(x, z))} \frac{\rho(d(x, y))}{\mu(B(x, 4 d(x, y)))}|f(y)| d \mu(y) \\
& \leq C_{1} \psi(2 d(x, z))\|f\|_{L^{(1, \varphi)}(G)} \\
& \int_{B(x, 2 d(x, z))} \frac{\rho(d(z, y))}{\mu(B(z, 4 d(z, y)))}|f(y)| d \mu(y) \\
& \leq \int_{B(z, 3 d(x, z)))} \frac{\rho(d(z, y))}{\mu(B(z, 4 d(z, y)))}|f(y)| d \mu(y) \\
& \leq C_{1}^{\prime} \psi(2 d(x, z))\|f\|_{L^{(1, \varphi)}(G)}
\end{aligned}
$$

for $x, z \in G$. On the other hand, we have by (52) and (59)

$$
\begin{aligned}
\int_{G \backslash B(x, 2 d(x, z))}\left|\frac{\rho(d(x, y))}{\mu(B(x, 4 d(x, y)))}-\frac{\rho(d(z, y))}{\mu(B(z, 4 d(z, y)))}\right| \\
\quad \times|f(y)| d \mu(y) \\
\leq C_{\rho}^{\prime} d(x, z)^{\theta} \int_{G \backslash B(x, 2 d(x, z))} \frac{\rho(d(x, y))}{\mu(B(x, 4 d(x, y))) d(x, y)^{\theta}} \\
\leq C_{2} d(x, z)^{\theta}\left(\int_{4 k_{1} d(x, z)}^{4 k_{2} d_{G}} \frac{\rho(t) \varphi(t)}{t^{1+\theta}} d t\right)\|f\|_{L^{(1, \varphi)}(G)} \\
\leq C_{2} \psi(2 d(x, z))\|f\|_{L^{(1, \varphi)}(G)} .
\end{aligned}
$$

Now from (89), (90), and (91), we establish

$$
\left|I_{\rho} f(x)-I_{\rho} f(z)\right| \leq C \psi(2 d(x, z))\|f\|_{L^{(1, \varphi)}(G)}
$$

for $x, z \in G$, as required.

\section{Appendix}

\section{A. Disproof of (15)}

Inequality (15) can be disproved in terms of Besov spaces and Triebel-Lizorkin spaces. Let $\psi \in C^{\infty}\left(\mathbb{R}^{n}\right)$ satisfy

$$
\chi_{B(0,4)} \leq \psi \leq \chi_{B(0,8)} .
$$

Define $T_{0} f:=\mathscr{F}^{-1}[\psi \cdot \mathscr{F} f]$ for $f \in \mathcal{S}^{\prime}\left(\mathbb{R}^{n}\right)$. For parameters $p \in(0, \infty)$ and $q \in(0, \infty)$ and for $f \in \mathcal{S}^{\prime}\left(\mathbb{R}^{n}\right)$, the Besov norm $\|\cdot\|_{B_{p, q}^{s}}$ and the Triebel-Lizorkin norm $\|\cdot\|_{F_{p, q}^{s}}$ are defined by

$$
\begin{gathered}
\|f\|_{B_{p, q}^{s}}:=\left\|T_{0} f\right\|_{L^{p}}+\left(\sum_{j=1}^{\infty}\left(2^{j s}\left\|S_{j} f\right\|_{L^{p}}\right)^{q}\right)^{1 / q}, \\
\|f\|_{F_{p, q}^{s}}:=\left\|T_{0} f\right\|_{L^{p}}+\left\|\left(\sum_{j=1}^{\infty}\left|2^{j s} S_{j} f\right|^{q}\right)^{1 / q}\right\|_{L^{p}},
\end{gathered}
$$

respectively, and for $p \in(0, \infty)$ and for $f \in \mathcal{S}^{\prime}\left(\mathbb{R}^{n}\right)$, the Besov norm $\|\cdot\|_{B_{p, \infty}^{s}}$ and the Triebel-Lizorkin norm $\|\cdot\|_{F_{p, \infty}^{s}}$ are defined by

$$
\begin{gathered}
\|f\|_{B_{p, \infty}^{s}}:=\left\|T_{0} f\right\|_{L^{p}}+\sup _{j \in \mathbb{N}} 2^{j s}\left\|S_{j} f\right\|_{L^{p}}, \\
\|f\|_{F_{p, \infty}^{s}}:=\left\|T_{0} f\right\|_{L^{p}}+\left\|\sup _{j \in \mathbb{N}}\left|2^{j s} S_{j} f\right|\right\|_{L^{p}} .
\end{gathered}
$$

Meanwhile, by denoting $\mathscr{P}\left(\mathbb{R}^{n}\right)$ the set of all polynomials, for parameters $p \in(1, \infty)$ and $q \in(1, \infty)$ and for a distribution $f \in \mathcal{S}^{\prime}\left(\mathbb{R}^{n}\right)$, the homogeneous Besov norm $\|\cdot\|_{\dot{B}_{p, q}^{s}}$ and the homogeneous Triebel-Lizorkin norm $\|\cdot\|_{\dot{F}_{p, q}^{s}}$ are defined 
by

$$
\begin{aligned}
\|f\|_{\dot{B}_{p, q}^{s}}:=\left(\sum_{j=-\infty}^{\infty}\left(2^{j s}\left\|S_{j} f\right\|_{L^{p}}\right)^{q}\right)^{1 / q}, \\
\|f\|_{\dot{F}_{p, q}^{s}}:=\left\|\left(\sum_{j=-\infty}^{\infty}\left|2^{j s} S_{j} f\right|^{q}\right)^{1 / q}\right\|_{L^{p}},
\end{aligned}
$$

respectively. Also, for $p \in(1, \infty)$ and $f \in \mathcal{S}^{\prime}\left(\mathbb{R}^{n}\right)$, one defines

$$
\begin{aligned}
& \|f\|_{\dot{B}_{p, \infty}^{s}}:=\sup _{j \in \mathbb{N}} 2^{j s}\left\|S_{j} f\right\|_{L^{p}}, \\
& \|f\|_{\dot{F}_{p, \infty}^{s}}:=\left\|\sup _{j \in \mathbb{N}}\left|2^{j s} S_{j} f\right|\right\|_{L^{p}},
\end{aligned}
$$

respectively.

It follows from (A.7) and (A.8) that

$$
\|f\|_{\dot{F}_{p, \infty}^{s}} \geq\|f\|_{\dot{B}_{p, \infty}^{s}} \quad\left(f \in \mathcal{S}^{\prime}\left(\mathbb{R}^{n}\right)\right) .
$$

Let $0<p, q \leq \infty$, and $s \in \mathbb{R}$. The inhomogeneous Besov space $B_{p, q}^{s}\left(\mathbb{R}^{n}\right)$ (resp. the homogeneous Besov space $\left.\dot{B}_{p, q}^{s}\left(\mathbb{R}^{n}\right)\right)$ is defined to be the set of all $f \in \mathcal{S}^{\prime}\left(\mathbb{R}^{n}\right)$ (resp. $f \in \mathcal{S}^{\prime}\left(\mathbb{R}^{n}\right) /$ $\mathscr{P}\left(\mathbb{R}^{n}\right)$ ) for which the norm $\|f\|_{B_{p, q}^{s}}$ (resp. $\|f\|_{\dot{B}_{p, q}^{s}}$ ) is finite, when $0<p, q \leq \infty$. Likewise, for $0<p<\infty, 0<q \leq \infty$ and $s \in \mathbb{R}$, the inhomogeneous Triebel-Lizorkin space $F_{p, q}^{s}\left(\mathbb{R}^{n}\right)$ (resp. the homogeneous Triebel-Lizorkin space $\dot{F}_{p, q}^{s}\left(\mathbb{R}^{n}\right)$ ) is defined to be the set of all $f \in \mathcal{S}^{\prime}\left(\mathbb{R}^{n}\right)$ (resp. $f \in \mathcal{S}^{\prime}\left(\mathbb{R}^{n}\right) /$ $\mathscr{P}\left(\mathbb{R}^{n}\right)$ ) for which the norm $\|f\|_{F_{p, q}^{s}}$ (resp. $\|f\|_{\dot{F}_{p, q}^{s}}$ ) is finite. To simplify the matters, even when we consider representatives in the function spaces $\dot{B}_{p, q}^{s}\left(\mathbb{R}^{n}\right)$ and $\dot{F}_{p, q}^{s}\left(\mathbb{R}^{n}\right)$, we forget that they are in equivalence classes, and we regard the function spaces $\dot{B}_{p, q}^{s}\left(\mathbb{R}^{n}\right)$ and $\dot{F}_{p, q}^{s}\left(\mathbb{R}^{n}\right)$ as subspaces of $\mathcal{S}^{\prime}\left(\mathbb{R}^{n}\right)$.

Keeping this in mind, let us disprove (15). We have

$$
\sup _{j \in \mathbb{Z}}\left\|S_{j} f\right\|_{L_{1, \lambda}} \leq c \sup _{j \in \mathbb{Z}}\left\|S_{j} f\right\|_{L^{n / \lambda}}=c\|f\|_{\dot{B}_{n / \lambda, \infty}^{0}} \leq c\|f\|_{\dot{F}_{n / \lambda, \infty}^{0}}
$$

from (5), (A.7), and (A.9).

However, according to [55, Theorem 11.2, (i), (11.2)], there exists $f \in F_{n / \lambda, \infty}^{0}\left(\mathbb{R}^{n}\right)$ such that it is not represented by $L_{\text {loc }}^{1}\left(\mathbb{R}^{n}\right)$-functions:

$$
f \in F_{n / \lambda, \infty}^{0}\left(\mathbb{R}^{n}\right) \backslash L_{\text {loc }}^{1}\left(\mathbb{R}^{n}\right) .
$$

If we consider $\mathscr{F}^{-1}[(1-\psi) \cdot \mathscr{F} f]$, where $\psi \in C_{\mathrm{c}}^{\infty}\left(\mathbb{R}^{n}\right)$ is from (A.1), we can arrange that $f \in F_{n / \lambda, \infty}^{0}\left(\mathbb{R}^{n}\right)$ can be chosen so that $\operatorname{supp}(\mathscr{F} f) \cap B(0,4)=\emptyset$. Indeed,

$$
\mathscr{F}^{-1}[\psi \cdot \mathscr{F} f] \in C^{\infty}\left(\mathbb{R}^{n}\right) .
$$

We suppose that the Fourier support of $f$ is away from $B(0,4)$. Let us admit that

$$
\|f\|_{\dot{F}_{n / \lambda, \infty}^{0}} \leq C\|f\|_{F_{n / \lambda, \infty}^{0}} \quad\left(f \in F_{n / \lambda, \infty}^{0}\left(\mathbb{R}^{n}\right)\right)
$$

under the understanding $\dot{F}_{n / \lambda, \infty}^{0}\left(\mathbb{R}^{n}\right) \subset S^{\prime}\left(\mathbb{R}^{n}\right)$. Note also that $L_{1, \lambda}\left(\mathbb{R}^{n}\right)$ is a subset of $L_{\text {loc }}^{1}\left(\mathbb{R}^{n}\right)$, hence our observation can be summarized as follows:

$$
L_{1, \lambda}\left(\mathbb{R}^{n}\right) \subset L_{\text {loc }}^{1}\left(\mathbb{R}^{n}\right), \quad f \in \dot{F}_{n / \lambda, \infty}^{0}\left(\mathbb{R}^{n}\right) \backslash L_{\text {loc }}^{1}\left(\mathbb{R}^{n}\right) .
$$

It then follows immediately that (15) fails since (15) implies

$$
L_{1, \lambda}\left(\mathbb{R}^{n}\right) \supset \dot{F}_{n / \lambda, \infty}^{0}\left(\mathbb{R}^{n}\right) .
$$

Inclusions (A.14) and (A.15) contradict obviously.

It remains to prove (A.13). Note that the frequency support of $f$ does not intersect with $B(0,4)$. Observe also that $\varphi\left(2^{-j}\right)$ has the frequency support in $B(0,4)$. Thus, we have $S_{j} f=\mathscr{F}^{-1}\left[\varphi\left(2^{-j}.\right) \mathscr{F} f\right]=0(j \leq-1)$, and hence

$$
\begin{aligned}
\|f\|_{\dot{F}_{n / \lambda, \infty}^{0}} & =\left\|\sup _{j \in \mathbb{Z}}\left|S_{j} f\right|\right\|_{L^{n / \lambda}}=\left\|\sup _{j \in \mathbb{N} \cup\{0\}}\left|S_{j} f\right|\right\|_{L^{n / \lambda}} \\
& \leq\|f\|_{F_{n / \lambda, \infty}^{0}}+\left\|S_{0} f\right\|_{L^{n / \lambda} .}
\end{aligned}
$$

Define

$$
W f:=\mathscr{F}^{-1}[\Psi \cdot \mathscr{F} f], \quad \Psi:=\frac{\varphi}{\psi+\varphi\left(2^{-1} \cdot\right)} .
$$

In view of the size of frequency support, we conclude $S_{0} f=$ $V T_{0} f+V S_{1} f$. Now we invoke the following Planchrel-PolyaNikolskii lemma.

Lemma A.1 (Planchrel-Polya-Nikols'kij [56, page 16]). Let $0<\eta \leq 1$. Assume that $f \in S^{\prime}\left(\mathbb{R}^{n}\right)$ has frequency support in $Q(0, R)$. Then, denoting by $M$ the Hardy-Littlewood maximal operator, we have

$$
\sup _{y \in \mathbb{R}^{n}} \frac{|f(x-y)|}{(1+R|y|)^{n / \eta}} \leq C M\left[|f|^{\eta}\right](x)^{1 / \eta},
$$

where $C$ is independent of $R>0$.

According to Lemma A.1 with $\eta=1 / 2$ and $R=16$, we conclude that

$$
\begin{aligned}
\left|S_{0} f(x)\right| \leq & C\left(\int_{\mathbb{R}^{n}}\left|\mathscr{F}^{-1} \Psi(y)\right| \cdot\left|T_{0} f(x-y)\right| d y\right. \\
& \left.\quad+\int_{\mathbb{R}^{n}}\left|\mathscr{F}^{-1} \Psi(y)\right| \cdot\left|S_{1} f(x-y)\right| d y\right) \\
\leq & C\left(M\left[\left|T_{0} f\right|^{1 / 2}\right](x)^{2}+M\left[\left|S_{1} f\right|^{1 / 2}\right](x)^{2}\right) \\
& \times \int_{\mathbb{R}^{n}}\left|\mathscr{F}^{-1} \Psi(y)\right|(1+|y|)^{2 n} d y \\
= & C\left(M\left[\left|T_{0} f\right|^{1 / 2}\right](x)^{2}+M\left[\left|S_{1} f\right|^{1 / 2}\right](x)^{2}\right),
\end{aligned}
$$

where for the last inequality, we invoked

$$
\left|\mathscr{F}^{-1} \Psi(y)\right| \leq \frac{C}{(1+|y|)^{3 n+1}} \quad\left(y \in \mathbb{R}^{n}\right) .
$$


By the fact that $\|F\|_{L^{n / \lambda}}=\|\sqrt{|F|}\|_{L^{2 n / \lambda}}^{2}$ and the $L^{2 n / \lambda}\left(\mathbb{R}^{n}\right)$ boundedness of the Hardy-Littlewood maximal operator, we conclude

$$
\begin{aligned}
\left\|S_{0} f\right\|_{L^{n / \lambda}} & \leq C\left(\left\|M\left[\left|T_{0} f\right|^{1 / 2}\right]\right\|_{L^{2 n / \lambda}}^{2}+\left\|M\left[\left|S_{1} f\right|^{1 / 2}\right]\right\|_{L^{2 n / \lambda}}^{2}\right) \\
& \leq C\left(\left\|\left|T_{0} f\right|^{1 / 2}\right\|_{L^{2 n / \lambda}}^{2}+\left\|\left|S_{1} f\right|^{1 / 2}\right\|_{L^{2 n / \lambda}}^{2}\right) \\
& =C\left(\left\|T_{0} f\right\|_{L^{n / \lambda}}+\left\|S_{1} f\right\|_{L^{n / \lambda}}\right) .
\end{aligned}
$$

Combining (A.16) and (A.21), we obtain the desired result.

\section{Acknowledgment}

The authors are grateful to Professor Victor Burenkov for his kind suggestion about the relations of various definitions of the Morrey norms.

\section{References}

[1] D. R. Adams, "A note on Riesz potentials," Duke Mathematical Journal, vol. 42, no. 4, pp. 765-778, 1975.

[2] V. I. Burenkov, V. S. Guliev, and G. V. Guliev, "Necessary and sufficient conditions for the boundedness of the Riesz potential in local Morrey-type spaces," Rossiǔskaya Akademiya Nauk, vol. 412, no. 5, pp. 585-589, 2007.

[3] V. I. Burenkov and V. S. Guliyev, "Necessary and sufficient conditions for the boundedness of the Riesz potential in local Morrey-type spaces," Potential Analysis, vol. 30, no. 3, pp. 211249, 2009.

[4] V. I. Burenkov, A. Gogatishvili, V. S. Guliyev, and R. Ch. Mustafayev, "Boundedness of the Riesz potential in local Morrey-type spaces," Potential Analysis, vol. 35, no. 1, pp. 67-87, 2011.

[5] V. I. Burenkov, "Recent progress in the problem of the boundedness of classical operators of real analysis," Eurasian Mathematical Journal, vol. 3, no. 3, pp. 8-27, 2012.

[6] F. Chiarenza and M. Frasca, "Morrey spaces and HardyLittlewood maximal function," Rendiconti di Matematica e delle sue Applicazioni, vol. 7, no. 7, pp. 273-279, 1987.

[7] Eridani, "On the boundedness of a generalized fractional integral on generalized Morrey spaces," Tamkang Journal of Mathematics, vol. 33, no. 4, pp. 335-340, 2002.

[8] Eridani, H. Gunawan, and E. Nakai, "On generalized fractional integral operators," Scientiae Mathematicae Japonicae, vol. 60, no. 3, pp. 539-550, 2004.

[9] H. Gunawan, "A note on the generalized fractional integral operators," Journal of the Indonesian Mathematical Society (MIHMI), vol. 9, no. 1, pp. 39-43, 2003.

[10] Y. Giga and T. Miyakawa, "Navier-Stokes flow in $\mathbb{R}^{3}$ with measures as initial vorticity and Morrey spaces," Communications in Partial Differential Equations, vol. 14, no. 5, pp. 577-618, 1989.

[11] K. Kurata, S. Nishigaki, and S. Sugano, "Boundedness of integral operators on generalized Morrey spaces and its application to Schrödinger operators," Proceedings of the American Mathematical Society, vol. 128, no. 4, pp. 1125-1134, 2000.

[12] E. Nakai, "Hardy-Littlewood maximal operator, singular integral operators and the Riesz potentials on generalized Morrey spaces," Mathematische Nachrichten, vol. 166, pp. 95-103, 1994.
[13] E. Nakai and H. Sumitomo, "On generalized Riesz potentials and spaces of some smooth functions," Scientiae Mathematicae Japonicae, vol. 54, no. 3, pp. 463-472, 2001.

[14] P. A. Olsen, "Fractional integration, Morrey spaces and a Schrödinger equation," Communications in Partial Differential Equations, vol. 20, no. 11-12, pp. 2005-2055, 1995.

[15] J. Peetre, "On the theory of $\mathscr{L}_{p, \lambda}$ spaces," Journal of Functional Analysis, vol. 4, pp. 71-87, 1969.

[16] S. Sugano and H. Tanaka, "Boundedness of fractional integral operators on generalized Morrey spaces," Scientiae Mathematicae Japonicae, vol. 58, no. 3, pp. 531-540, 2003.

[17] N. S. Trudinger, "On imbeddings into Orlicz spaces and some applications," vol. 17, pp. 473-483, 1967.

[18] J. Serrin, "A remark on Morrey potential," Contemporary Math, vol. 426, pp. 307-315, 2007.

[19] Y. Mizuta, E. Nakai, T. Ohno, and T. Shimomura, "Boundedness of fractional integral operators on Morrey spaces and Sobolev embeddings for generalized Riesz potentials," Journal of the Mathematical Society of Japan, vol. 62, no. 3, pp. 707-744, 2010.

[20] Y. Mizuta, E. Nakai, T. Ohno, and T. Shimomura, "An elementary proof of Sobolev embeddings for Riesz potentials of functions in Morrey spaces $L^{1, \nu, \beta}(G)$," Hiroshima Mathematical Journal, vol. 38, no. 3, pp. 425-436, 2008.

[21] E. Nakai, "On generalized fractional integrals on the weak Orlicz spaces, $B M O_{\varphi}$, the Morrey spaces and the Campanato spaces," in Function Spaces, Interpolation Theory and Related Topics (Lund, 2000), pp. 389-401, de Gruyter, Berlin, Germany, 2002.

[22] H. Gunawan, E. Nakai, Y. Sawano, and H. Tanaka, "Generalized stummel class and morrey spaces," PubLications De L'Institut Mathematique, vol. 92, no. 106, pp. 127-138, 2012.

[23] Y. Sawano and S. Shirai, "Compact commutators on Morrey spaces with non-doubling measures," Georgian Mathematical Journal, vol. 15, no. 2, pp. 353-376, 2008.

[24] Y. Sawano and H. Tanaka, "Sharp maximal inequalities and commutators on Morrey spaces with non-doubling measures," Taiwanese Journal of Mathematics, vol. 11, no. 4, pp. 1091-1112, 2007.

[25] Y. Sawano and H. Tanaka, "The John-Nirenberg type inequality for non-doubling measures," Studia Mathematica, vol. 181, no. 2, pp. 153-170, 2007.

[26] C. Fefferman and E. M. Stein, " $H^{p}$ spaces of several variables," Acta Mathematica, vol. 129, no. 3-4, pp. 137-193, 1972.

[27] Y. Sawano, "Generalized Morrey spaces for non-doubling measures," Nonlinear Differential Equations and Applications, vol.15, no. 4-5, pp. 413-425, 2008.

[28] M. Izuki, Y. Sawano, and H. Tanaka, "Weighted BesovMorrey spaces and Triebel-Lizorkin spaces, Harmonic analysis and nonlinear partial differential equations, 21-60," RIMS Kôkyûroku Bessatsu B22, Mathematical Sciences Research Institute (RIMS), Kyoto, Japan, 2010.

[29] H. Kozono and M. Yamazaki, "Semilinear heat equations and the Navier-Stokes equation with distributions in new function spaces as initial data," Communications in Partial Differential Equations, vol. 19, no. 5-6, pp. 959-1014, 1994.

[30] A. L. Mazzucato, "Decomposition of Besov-Morrey spaces," in Harmonic Analysis at Mount Holyoke, vol. 320 of Contemporary Mathematics, pp. 279-294, American Mathematical Society, Providence, RI, USA, 2003.

[31] A. L. Mazzucato, "Besov-Morrey spaces: function space theory and applications to non-linear PDE," Transactions of the American Mathematical Society, vol. 355, no. 4, pp. 1297-1364, 2003. 
[32] Y. Sawano, "Brézis-Gallouët-Wainger type inequality for BesovMorrey spaces," Studia Mathematica, vol. 196, no. 1, pp. 91-101, 2010.

[33] Y. Sawano, "Besov-Morrey spaces and Triebel-Lizorkin-Morrey spaces on domains," Mathematische Nachrichten, vol. 283, no. 10, pp. 1456-1487, 2010.

[34] Y. Sawano and H. Tanaka, "Decompositions of Besov-Morrey spaces and Triebel-Lizorkin-Morrey spaces," Mathematische Zeitschrift, vol. 257, no. 4, pp. 871-905, 2007.

[35] Y. Sawano and H. Tanaka, "Besov-Morrey spaces and TriebelLizorkin-Morrey spaces for non-doubling measures," Mathematische Nachrichten, vol. 282, no. 12, pp. 1788-1810, 2009.

[36] L. Tang and J. Xu, "Some properties of Morrey type BesovTriebel spaces," Mathematische Nachrichten, vol. 278, no. 7-8, pp. 904-917, 2005.

[37] W. Yuan, Y. Sawano, and D. Yang, "Decompositions of BesovHausdorff and Triebel-Lizorkin-Hausdorff spaces and their applications," Journal of Mathematical Analysis and Applications, vol. 369, no. 2, pp. 736-757, 2010.

[38] W. Yuan, W. Sickel, and D. Yang, Morrey and Campanato Meet Besov, Lizorkin and Triebel, vol. 2005 of Lecture Notes in Mathematics, Springer, Berlin, Germany, 2010.

[39] D. Yang and W. Yuan, "A new class of function spaces connecting Triebel-Lizorkin spaces and $Q$ spaces," Journal of Functional Analysis, vol. 255, no. 10, pp. 2760-2809, 2008.

[40] D. Yang and W. Yuan, "New Besov-type spaces and TriebelLizorkin-type spaces including Q spaces," Mathematische Zeitschrift, vol. 265, no. 2, pp. 451-480, 2010.

[41] D. Yang and W. Yuan, "Characterizations of Besov-type and Triebel-Lizorkin-type spaces via maximal functions and local means," Nonlinear Analysis, vol. 73, no. 12, pp. 3805-3820, 2010.

[42] D. Yang and W. Yuan, "Dual properties of Triebel-Lizorkin-type spaces and their applications," Zeitschrift für Analysis und ihre Anwendungen, vol. 30, no. 1, pp. 29-58, 2011.

[43] D. Yang, W. Yuan, and C. Zhuo, "Fourier multipliers on Triebel-Lizorkin-type spaces," Journal of Function Spaces and Applications, vol. 2012, Article ID 431016, 37 pages, 2012.

[44] Y. Sawano and T. Shimomura, "Sobolev embeddings for Riesz potentials of functions in non-doubling Morrey spaces of variable exponents," Collectanea Mathematica, 2013.

[45] Y. Sawano, " $l^{q}$-valued extension of the fractional maximal operators for non-doubling measures via potential operators," International Journal of Pure and Applied Mathematics, vol. 26, no. 4, pp. 505-523, 2006.

[46] E. Nakai, "A characterization of pointwise multipliers on the Morrey spaces," Scientiae Mathematicae, vol. 3, no. 3, pp. 445454, 2000.

[47] Y. Sawano, S. Sugano, and H. Tanaka, "Generalized fractional integral operators and fractional maximal operators in the framework of Morrey spaces," Transactions of the American Mathematical Society, vol. 363, no. 12, pp. 6481-6503, 2011.

[48] Y. Sawano and H. Tanaka, "Morrey spaces for non-doubling measures," Acta Mathematica Sinica, vol. 21, no. 6, pp. 15351544, 2005.

[49] Eridani and Y. Sawano, "Fractional integral operators in generalized Morrey spaces defined on metric measure spaces," Proceedings of A. Razmadze Mathematical Institute, vol. 158, pp. 13-24, 2012.

[50] E. Nakai, "Generalized fractional integrals on Orlicz-Morrey spaces," in Banach and Function Spaces, pp. 323-333, Yokohama Publishers, Yokohama, Japan, 2004.
[51] E. Nakai, "Orlicz-Morrey spaces and the Hardy-Littlewood maximal function," Studia Mathematica, vol. 188, no. 3, pp. 193221, 2008.

[52] E. Nakai and Y. Sawano, "Hardy spaces with variable exponents and generalized Campanato spaces," Journal of Functional Analysis, vol. 262, no. 9, pp. 3665-3748, 2012.

[53] S. Nagayasu and H. Wadade, "Characterization of the critical Sobolev space on the optimal singularity at the origin," Journal of Functional Analysis, vol. 258, no. 11, pp. 3725-3757, 2010.

[54] Y. Sawano, S. Sugano, and H. Tanaka, "Orlicz-Morrey spaces and fractional operators," Potential Analysis, vol. 36, no. 4, pp. 517-556, 2012.

[55] H. Triebel, The Structure of Functions, vol. 97 of Monographs in Mathematics, Birkhäuser, Basel, Switzerland, 2001.

[56] H. Triebel, Theory of Function Spaces, vol. 78 of Monographs in Mathematics, Birkhäuser, Basel, Switzerland, 1983. 


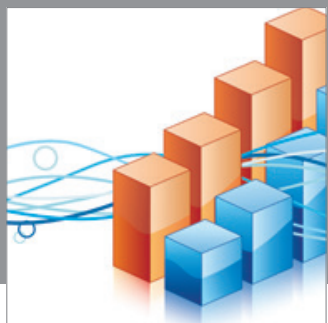

Advances in

Operations Research

mansans

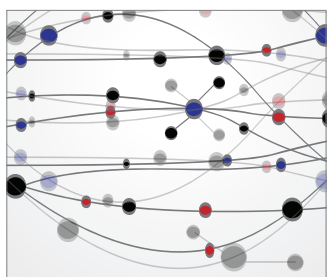

The Scientific World Journal
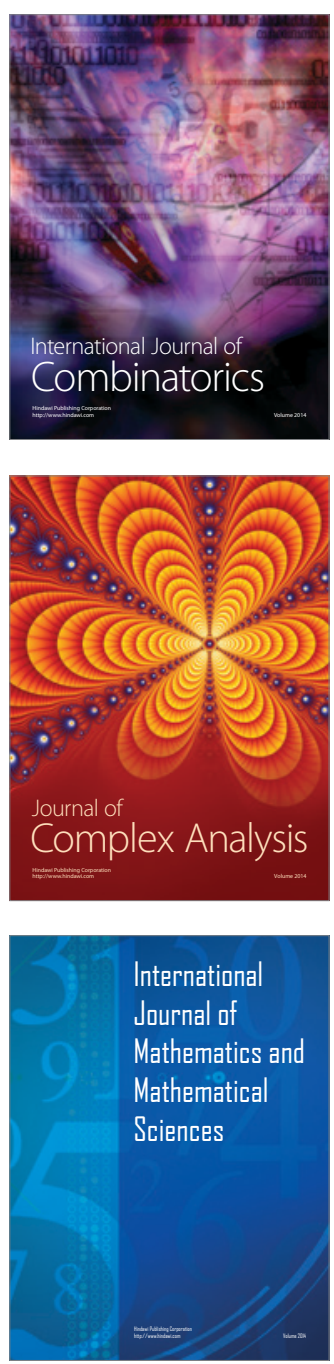
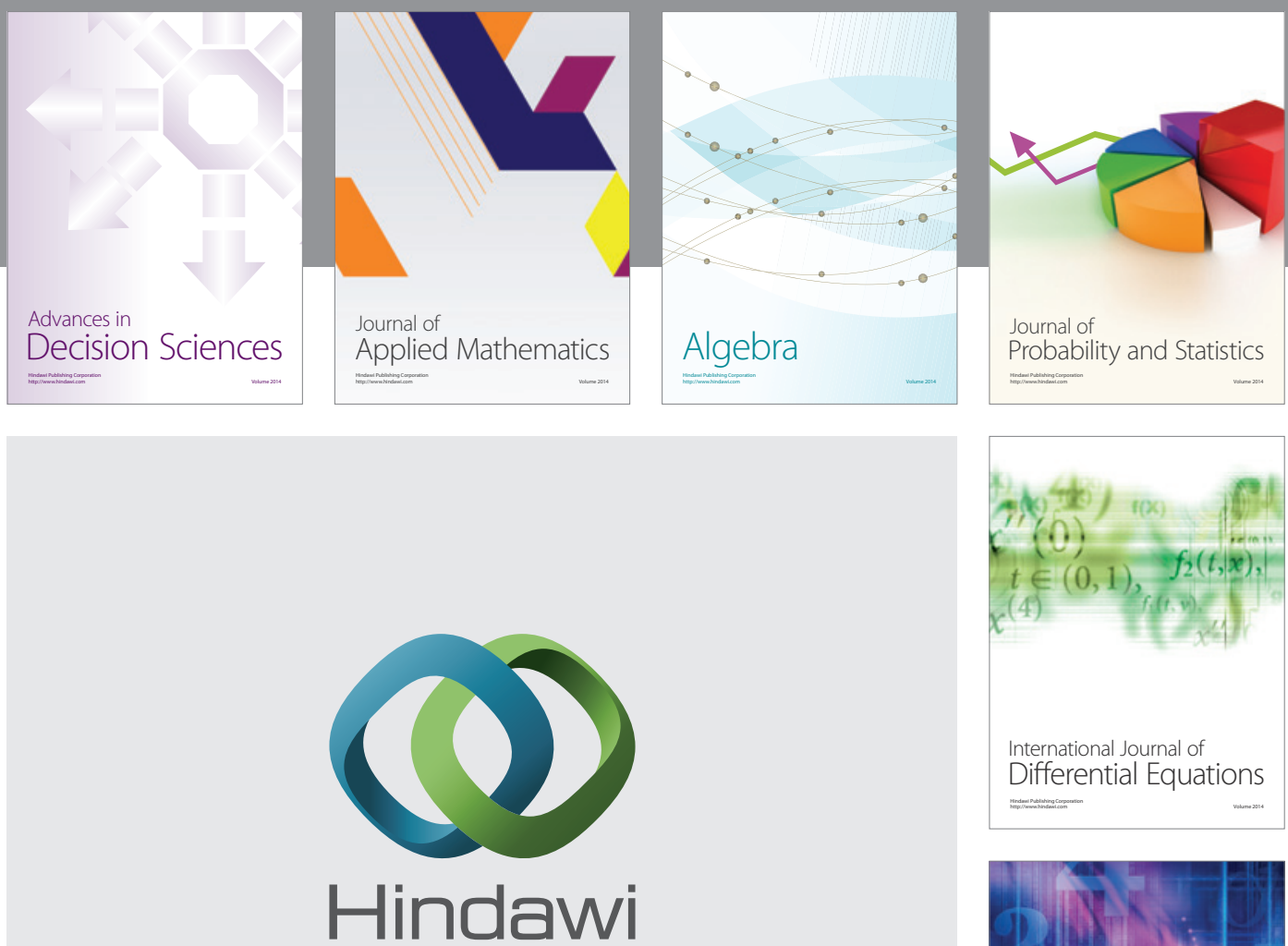

Submit your manuscripts at http://www.hindawi.com
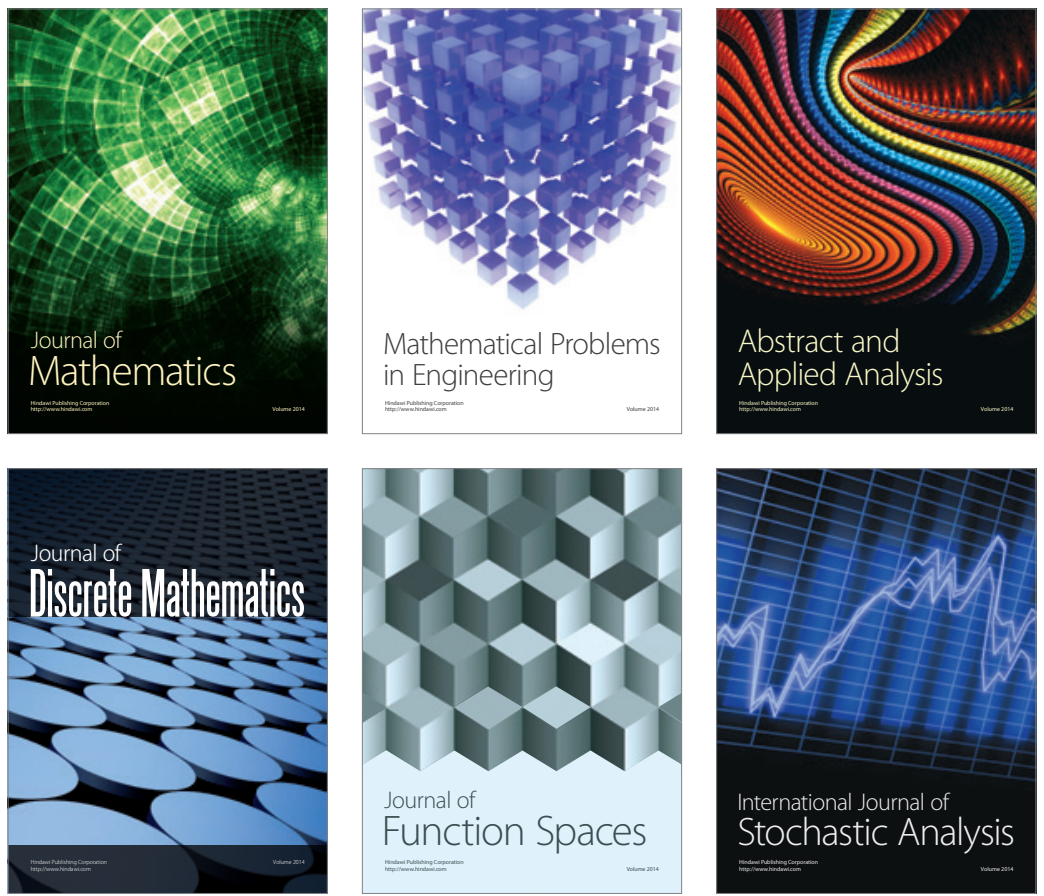

Journal of

Function Spaces

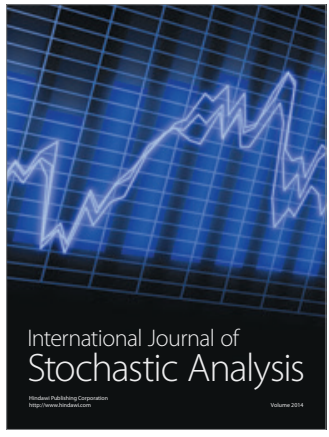

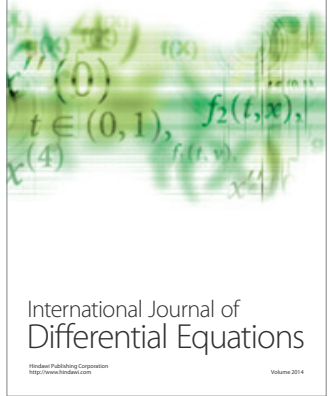
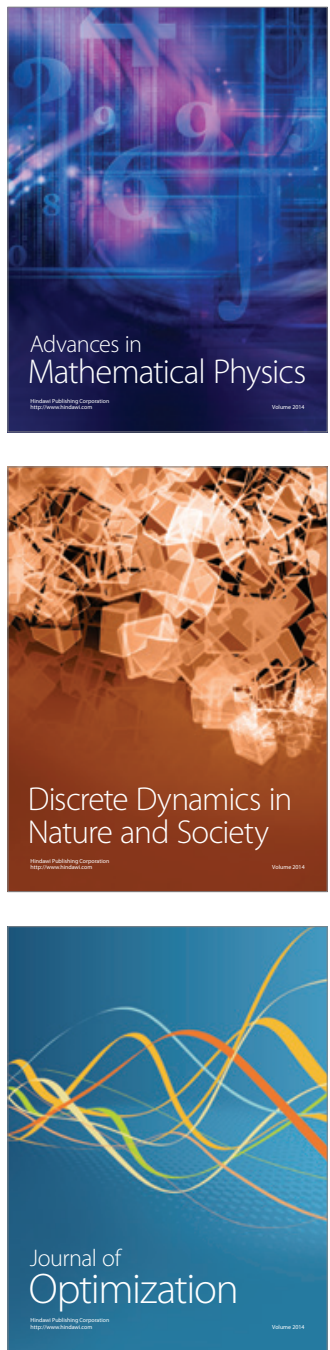